\title{
Uso do espaço por Lontra longicaudis (Mustelidae, Carnivora) em ambiente alterado no rio Caí, Rio Grande do Sul, Brasil
}

\author{
Luciane D. Coletti ${ }^{1}$, Thaís Michel ${ }^{1}$, Daniela Sanfelice ${ }^{2} \&$ Márcia M. A. Jardim ${ }^{1}$
}

1. Laboratório de Mastozoologia, Museu de Ciências Naturais, Fundação Zoobotânica do Rio Grande do Sul. Rua Dr. Salvador França, 1427, Jardim Botânico, 90690-000 Porto Alegre, RS, Brasil.
(lucoletti@gmail.com)
2. Instituto Federal de Educação, Ciência e Tecnologia do Rio Grande do Sul, Campus Osório. Rua Machado de Assis, 1456, Sulbrasileiro, 95520-000 Osório, RS, Brasil. (daniela.sanfelice@gmail.com)

ABSTRACT. Spatial habitat use by Lontra longicaudis (Mustelidae, Carnivora) in an anthropically modified environment in Caí river, Rio Grande do Sul , Brazil. The use of shelters and space by the Neotropical otter, Lontra longicaudis (Olfers, 1818), was studied in an environment formerly and presently altered by human presence in the Caí river, Triunfo, RS. Most marking sites were used occasionally, while a small percentage was frequently and intensely used. Most sprainting was observed on the soil, and sites were, on average, $1.65 \mathrm{~m}$ away from the water level. In total, seven shelters were found in the study area and the otter showed preference for specific ones. The most used shelter was a holt built in the riverbank under the roots of a tree. This was the largest shelter and the only one presenting underground galleries. The other shelters were above ground, amongst heaps of branches beneath the vegetation, or under roots and/or fallen tree trunks. The use of space by the otter was correlated to the location of the shelters and to the level of vegetation cover, and seems to be poorly influenced by human disturbance. For the Neotropical otter conservancy we indicate the importance of maintaining the riparian buffers and the protection of the areas with the presence of shelters

KEYWORDS. Neotropical otter, scent marking, shelters, sprainting sites.

RESUMO. A utilização de abrigos e do espaço pela lontra neotropical (Lontra longicaudis) (Olfers, 1818), foi estudada em um ambiente alterado e com presença humana no rio Caí, Triunfo, RS. A maioria dos sítios de marcação teve uso ocasional, e uma pequena percentagem teve uso frequente e intenso. A maioria das marcações de cheiro se encontrava no solo a uma média de distância de $1,65 \mathrm{~m}$ da linha d'água. Ao todo foram encontrados sete abrigos na área de estudo, sendo que a lontra demonstrou preferência por abrigos específicos. O abrigo mais utilizado se constituía em escavações na barranca do rio sob as raízes de uma árvore, sendo este o de maiores dimensões e o único com galerias sob o solo. Os demais abrigos se encontravam no nível do solo e consistiam em emaranhados de galhos sob a vegetação, ou de raízes e/ou troncos caídos. O uso do espaço pela lontra esteve correlacionado à localização de suas tocas, ao grau de cobertura vegetal do local e parece ter sido pouco influenciado pelo distúrbio humano. Para medidas de conservação da lontra neotropical ressalta-se a importância da manutenção da mata ciliar e a proteção das áreas com a presença de abrigos.

PALAVRAS-CHAVE. Lontra neotropical, marcação de cheiro, abrigos, sítios de marcação.

A lontra neotropical Lontra longicaudis (Olfers, 1818) é um mustelídeo semiaquático que possui ampla distribuição geográfica, desde o México até o norte da Argentina, ocorrendo em praticamente todo Brasil (EMmONs \& FeER, 1997; EISENBERG \& REDFORD, 1999; Waldemarin \& Alvarez, 2008). Possui o hábito de demarcar o território com fezes e muco anal, que servem como marcações de cheiro dentro de seu território e indicam sua presença no local (PARDINI \& TRAJANO, 1999; Waldemarin \& Colares, 2000; Quadros \& MonteIro-Filho, 2002). Estas marcações têm sido registradas em diferentes locais de deposição dentro de seu território, podendo ser encontradas tanto em locais conspícuos como rochas, matacões e montes de terra, como no solo e no interior dos abrigos (Soldateli \& Blacher, 1996; PARDini \& TraJano, 1999; WALDEMARIN \& Colares, 2000; Quadros \& Monteiro-Filho, 2002; Alarcon \& Simões-Lopes, 2003; KASPER et al., 2004, 2008; Quintela et al., 2011).

Da mesma forma, há diferentes tipos de abrigos que foram utilizados pela lontra no Brasil, sendo que sua constituição pode variar de escavações nas margens de rios e sob raízes de árvores, de espaços entre emaranhados de vegetação fechada, até galerias sob rochas e matacões (SOldateli \& Blacher, 1996; Pardini \& Trajano, 1999; Quadros \& Monteiro-
Filho, 2002; Waldemarin \& Colares, 2000; Alarcon \& SimÕes-Lopes, 2003; KASPER et al., 2004, 2008; QUinTEla et al., 2011).

Apesar dos vários estudos já realizados no país, poucos foram os que analisaram o uso do espaço pela espécie em locais degradados (PARDINI \& TRAJANO, 1999; SOlDATEli \& Blacher, 1996; AlarCon \& SimÕes-Lopes, 2003; KASPER et al., 2008; QUADROS, 2012) ou o impacto da presença humana e da degradação ambiental sobre a espécie (Alarcon \& Simões-Lopes, 2003; KasPer et al., 2008; Quadros, 2012; Quintela et al., 2012). Lontra longicaudis é considerada versátil, podendo alterar alguns aspectos de seu comportamento de acordo com o ambiente em que habita, inclusive tolerando a influência humana em alguns locais (PARDINI \& TRAJANO, 1999; Alarcon \& Simões-Lopes, 2003; QuAdros, 2012).

As principais ameaças à lontra são o desmatamento, a contaminação e a poluição dos cursos d'água, a caça, as atividades de agricultura e o barramento e mineração nos rios (Waldemarin \& Alvarez, 2008). A espécie é suspeita de estar ameaçada de extinção, no entanto, como faltam informações acerca do tamanho de suas populações e de sua distribuição atual, além do efeito cumulativo destas ameaças ao longo de sua distribuição, ela é considerada como dados insuficientes (DD) a nível mundial (IUCN, 2013). 
Dentro deste contexto, é importante o estudo de $L$. longicaudis em ambientes antropizados, a fim de se obter maiores informações sobre o impacto destas ameaças sobre a espécie. O presente trabalho objetivou avaliar de forma indireta o comportamento de L. longicaudis em um trecho de rio antropizado e com presença humana, através de suas marcações de cheiro. As hipóteses a serem investigadas foram se as lontras utilizam de forma uniforme o ambiente ou apresentam um comportamento de uso do espaço mais intenso ao redor de seus abrigos, em locais protegidos e com maior grau de cobertura vegetal.

\section{MATERIAL E MÉTODOS}

O trabalho foi desenvolvido no curso inferior do rio Caí, município de Triunfo, Rio Grande do Sul, Brasil $\left(29^{\circ} 44^{\prime}\right.$ e $29^{\circ} 55^{\prime} \mathrm{S} ; 51^{\circ} 21^{\prime}$ e $\left.51^{\circ} 28^{\prime} \mathrm{W}\right)$. O rio Caí é o principal corpo d'água da bacia hidrográfica de mesmo nome, cuja abrangência atinge 42 municípios com aproximadamente 1,2 milhão de habitantes. Em seu curso inferior, o rio se encontra intensamente antropizado, com a qualidade da água comprometida pelo lançamento de efluentes de origem doméstica (esgotos), industrial (efluentes industriais) e rural (agrotóxicos e dejetos animais). As margens também são alteradas pela retirada de areia e da vegetação marginal para cultivos ou pastagens (FEPAM, 2010).

O trabalho foi realizado em uma extensão de aproximadamente $1000 \mathrm{~m}$ ao longo do rio; neste trecho o terreno é plano, formado por várzeas e em alguns pontos por barrancos de terra baixos nas margens (OliveIRA et al., 2010). Parte da área na margem leste integra um parque particular de 68 ha denominado Estação Ambiental Braskem, área de proteção ambiental inserida no complexo industrial do Pólo Petroquímico do Sul. A margem oeste pertence a propriedades particulares, com predomínio de sítios para criação de gado. O local sofre interferência antrópica com fluxo frequente de pessoas nas margens e de pescadores ao longo do rio, onde também é realizado o transporte de materiais (e.g. areia, seixos, cascalho, calcário) através de barcaças.

A vegetação local se caracteriza como Floresta Estacional Decidual Aluvial (IBGE, 1986), composta principalmente por maricás (Mimosa binucronata Kuntze), ingazeiros (Inga uruguensis Willd), acáciade-flores-vermelhas (Sesbania punicea Benth), sarandi (Sebatiania schottiana Müll. Arg.) e cipó-unha-degato (Macfadyena unguis-cati L. G. Lohmann) e ainda várias espécies introduzidas. Do lado do parque há predomínio da vegetação nativa em regeneração (Veitenheimer-Mendes et al., 2000) e, na margem oposta, mesmo mantida a vegetação ripária, o restante da área foi alterado com pastagens e plantações de arroz. O clima do local é subtropical úmido ("Cfa" segundo Köppen). Os dados de temperatura e precipitação foram obtidos junto ao $8^{\circ}$ Distrito de Meteorologia do Instituto
Nacional de Meteorologia do Ministério da Agricultura e do Abastecimento, sendo a temperatura média nos anos amostrados de $20^{\circ} \mathrm{C}$ e a temperatura mínima mensal de $8,1^{\circ} \mathrm{C}$ e a máxima mensal $32,1^{\circ} \mathrm{C}$. A precipitação mensal média para os anos amostrados foi $118 \mathrm{~mm}$, sendo 30,3 $\mathrm{mm}$ e $202,2 \mathrm{~mm}$ a menor e a maior precipitação mensal média, respectivamente.

Foram realizadas 16 expedições de janeiro de 2006 a dezembro de 2007. Não foi possível inventariar todos os meses de cada ano devido às cheias recorrentes no rio Caí (Oliveira et al., 2010), que submergiam a área de estudo e todos os abrigos monitorados.

A cada mês amostrado o trecho foi percorrido a pé em ambas as margens à procura de vestígios de lontras tais como marcações de cheiro, sítios de deposição, abrigos, rastros e arranhões em um transecto de $10 \mathrm{~m}$ de largura em cada margem. Como marcação de cheiro, foram consideradas fezes ou muco anal encontrados na área de estudo (KASPER et al., 2004). Sítio de deposição foi definido de acordo com QuADros \& MonteIroFilho (2002) como sendo um abrigo, árvore caída ou área do solo onde foi encontrada ao menos uma marcação de cheiro ao longo do estudo. Abrigos foram considerados cavidades nas margens do rio ou espaços dentro da vegetação densa com o chão limpo ou arranhado que contivessem pegadas ou fezes de lontra (Pardini \& Trajano, 1999).

Para cada vestígio e abrigo encontrado, foi registrado o local com GPS (Garmim $\left.{ }^{\circledR} 12\right)$ e a distância da linha d'água mensurada com trena métrica. Se as marcações de cheiro eram encontradas em troncos acima do solo, foi medida também a distância desta em relação ao solo. Todas as marcações de cheiro encontradas foram removidas para evitar recontagem (PARDINI \& TRAJANO, 1999). A estrutura de todos os abrigos foi descrita e esses foram monitorados quanto à sua utilização (Pardini \& Trajano, 1999), sendo o principal indício de reutilização a presença de fezes ou pegadas em seu interior (KASPER et al., 2004). Para todos os sítios de marcação foi monitorada a sua reutilização através da presença de marcações de cheiro (PARDINI \& TRAJANO, 1999; Quadros \& Monteiro-Filho, 2002; KasPer et al., 2008). A utilização de marcações de cheiro como medida da intensidade de uso foi utilizada baseada em diversos trabalhos com outras espécies de lontras, em que se demonstrou ser um bom indicador da intensidade de uso de áreas específicas e do uso do hábitat (MASON \& MacDonald, 1987; Guter et al., 2008).

Os sítios de marcação e os abrigos foram classificados em três categorias distintas, de acordo com o seu uso: de uso ocasional (1 a 3 meses de utilização), de uso frequente (de 4 a 7 meses) e de uso intenso (de 8 a 11 meses de utilização), modificado de QUADRos \& Monteiro-Filho (2002) e Pardini \& Trajano (1999).

A análise do uso do espaço foi realizada utilizando uma janela de Imagem de satélite Quickbird e pontos de georreferenciamento do Google Earth (data de 
04/04/2006). Para o parcelamento do trecho monitorado, foram geradas 104 quadrículas de 50 X 50 m no software ArcGIS (Fig. 1). O mapa de uso e cobertura da terra e os recursos hídricos foram gerados por classificação visual em tela. Também no ArcGIS foram utilizadas as funções union para unir os temas vegetação e recursos d'água para cada quadrícula e join para junção das quadrículas com os pontos de ocorrência de lontras. As quadrículas localizadas inteiramente na água foram descartadas, totalizando 101 quadrículas. Os sítios de marcação nestas áreas correspondiam a galhos inclinados sobre o leito do rio e para fins das análises subsequentes estas não se aplicam.
Para testar a correlação entre a existência de abrigos na quadrícula e o número de marcações depositadas, foi utilizado o Coeficiente de Correlação de Spearman no SPSS 16, similar ao efetuado por QUADROS (2012). Da mesma forma, foi testada a correlação entre a cobertura vegetal da quadrícula e o número de marcações depositadas.

Para testar se houve maior utilização pela lontra da margem de rio onde se situa uma área de proteção particular em relação à margem sem proteção, foi utilizado o Teste de Mann-Withney (SPSS 16) para comparar o número de marcações de cheiro entre as quadrículas das duas margens.

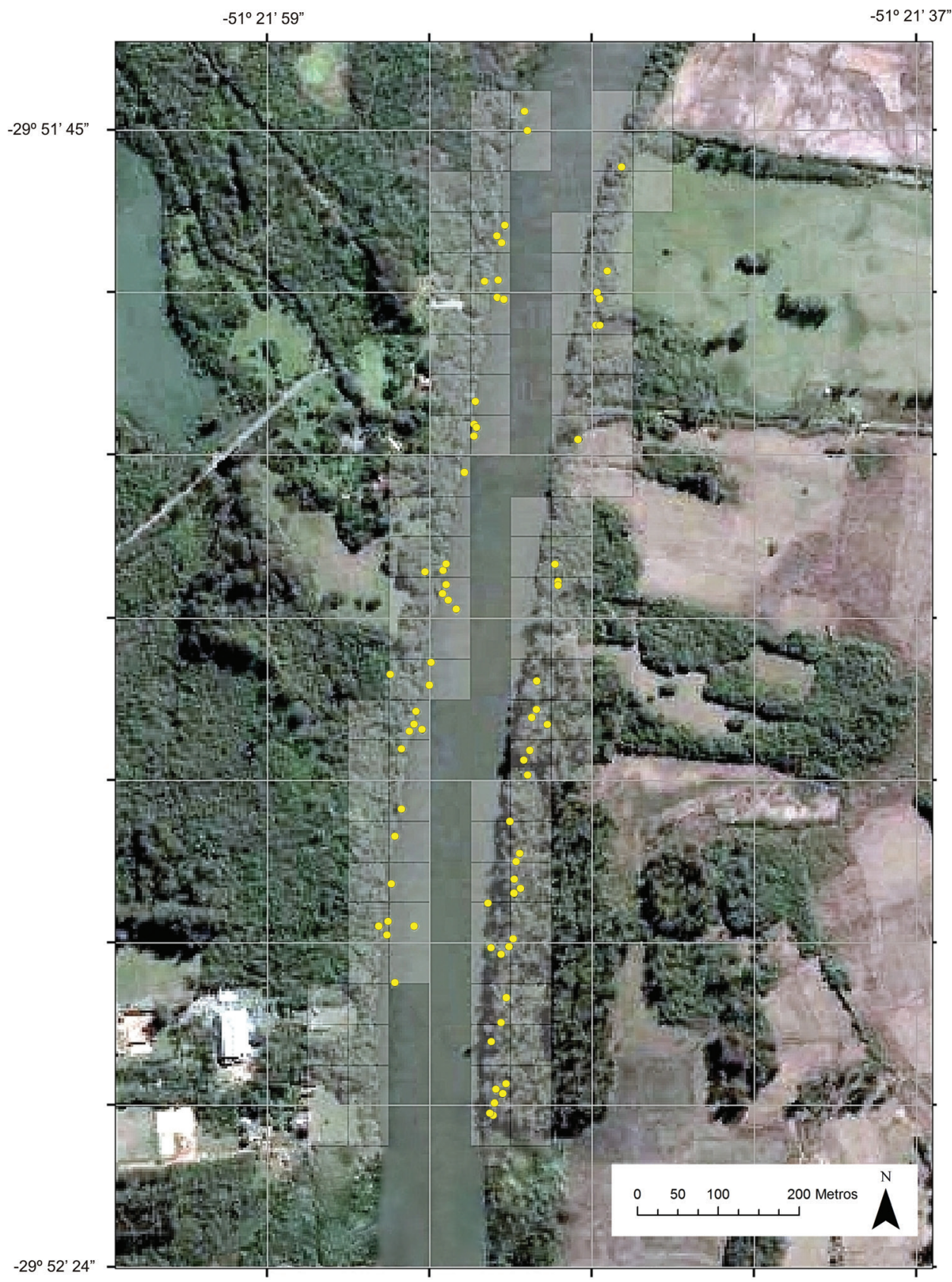

Fig. 1. Localização da área com o trecho do rio amostrado com as quadrículas de 50x50m e os pontos (em amarelo) com a localização dos vestígios e abrigos utilizados por Lontra longicaudis no período de janeiro de 2006 a dezembro de 2007, no curso inferior do rio Caí, Triunfo, Rio Grande do Sul. 


\section{RESULTADOS}

Foram encontrados 226 vestígios durante todo período de estudo. As marcações de cheiro foram depositadas em 75 sítios de demarcação. A maioria dos sítios de marcação teve uso ocasional ( $69 \%, \mathrm{n}=52), 20 \%$ foram de uso frequente $(n=15)$ e uma baixa percentagem dos sítios foi utilizado de forma intensa $(11 \%, n=8)$. A maior parte dos sítios de marcação apresentou reutilização ao longo do período de estudo $(51 \%$; $n=38)$; no entanto, uma grande percentagem $(49 \% ; n=37)$ foi utilizada apenas uma vez. A maioria das marcações de cheiro se encontrava no solo ( $\mathrm{n}=144)$ e a uma distância média de $1,65 \mathrm{~m}$ da linha d'água $(\mathrm{n}=140 ; \mathrm{dp}=1,18)$.

Ao todo, foram encontrados sete abrigos em utilização na área de estudo e em apenas uma amostragem não foi registrado o uso de tais abrigos. $\mathrm{O}$ abrigo mais utilizado apresentou sinais de presença da lontra em $69 \%$ das coletas $(\mathrm{n}=11)$, sendo considerado de uso intenso. Era uma cavidade natural escavada pela ação da erosão da água embaixo das raízes de uma árvore, e possuía as maiores dimensões dentre os abrigos estudados (em torno de 1,5 $\mathrm{m}$ de altura, $3 \mathrm{~m}$ de largura e $2 \mathrm{~m}$ de profundidade). As dimensões deste abrigo foram diferentes ao longo do estudo devido à ação da erosão efetuada pelo rio e constantes escavações da lontra.

A maioria dos abrigos consistia de galerias sob emaranhados de galhos ou sob raízes e troncos caídos, com o solo exposto e contendo em certas ocasiões arranhões efetuados pela lontra. Dentre estes, dois foram considerados de uso intenso, tendo sido encontrados sinais de utilização em nove e oito amostragens respectivamente (56\% e $50 \%$ de frequência de uso). Houve ainda um abrigo com uso frequente, com seis meses de utilização (38\% de frequência de uso). Os três abrigos restantes tiveram uma média de visitação de duas amostragens e com média de visitação de 13\%, sendo considerados de uso ocasional.

Não houve diferença na utilização de sítios de marcação entre a margem onde se situa uma área de proteção particular em relação à margem onde não há proteção $(U=1207 ; p>0,05 ; n=101)$. Foi verificada correlação positiva de média intensidade entre a presença de abrigos na quadrícula e o número de marcações de cheiro depositadas $\left(\mathrm{r}_{\mathrm{s}}=0,37 ; \mathrm{p}<0,001 ; \mathrm{n}=101\right)$, assim como foi registrada correlação positiva de baixa intensidade entre a cobertura vegetal da quadrícula e o número de marcações de cheiro depositadas $\left(\mathrm{r}_{\mathrm{s}}=0,26\right.$; $\mathrm{p}$ $<0,009 ; \mathrm{n}=101)$.

\section{DISCUSSÃO}

A lontra utilizou como locais de deposição o solo, troncos caídos e galhos e, sobretudo, os abrigos, sendo que este padrão tem sido encontrado em rios semelhantes ao trecho do rio Caí estudado, com margens argilosas e sem formações rochosas (Quadros \& MonTeIro-
FiLHO, 2002; KASPER et al., 2004). No rio Caí Lontra longicaudis reutilizou a maioria dos sítios de marcação encontrados, sendo que vários foram frequentemente utilizados. A reutilização de sítios de marcação tem sido verificada para $L$. longicaudis (QUADRos \& MONTEIROFilHo, 2002; KASPER et al., 2008; QUINTELA et al., 2011). O comportamento de marcação que as lontras apresentam é relacionado à defesa de território e à sinalização odorífera entre os indivíduos (MASON \& MacDonald, 2009; Oldham \& Black, 2009). Desta forma, a reutilização dos sítios de marcação pode ser vista como uma forma de reforçar a sinalização em áreas-chave do território, sendo mais intensa nos centros de atividade (Quadros \& Monteiro-Filho, 2002).

A conformação dos abrigos encontrados na área estudada - cavidades naturais ou galerias em meio ou abaixo de raízes e troncos - é similar à registrada em rios que formam barrancos de terra e várzeas nas margens (Waldemarin \& Colares, 2000; Quadros \& MonteIro-FilHo, 2002; KASPER et al., 2004, 2008; Quintela et al., 2011), que é distinta da observada em rios onde as margens são rochosas, onde a maior parte dos abrigos se constitui em espaços formados entre as rochas (PARDini \& Trajano, 1999; KASPER et al., 2004). Entretanto, diferente do encontrado em outros locais, onde a maioria dos abrigos é escavada sob o solo (Quadros \& Monteiro-Filho, 2002; Kasper et al., 2008; Quintela et al., 2011), os abrigos mais comuns na área foram aqueles que se encontravam acima do solo, sem escavações feitas pela lontra. Este padrão também foi verificado por WALDEMARIN \& COLARES (2000), que relacionaram tal comportamento com o fato da área possuir baixa perturbação. No entanto, na área aqui estudada isso não poderia explicar a conformação dos abrigos, já que existe distúrbio com constante presença humana. É possível que a lontra utilize locais acima do solo em meio à vegetação densa ou a troncos e galhos caídos, como uma alternativa para se abrigar, em vista da escassez de cavidades naturais que possam ser utilizadas como abrigo no local de estudo. Sendo assim, os abrigos encontrados acima do solo seriam apenas um reflexo da capacidade de adaptação da lontra aos diferentes tipos de conformação das margens dos rios. Esta plasticidade de Lontra longicaudis na utilização de abrigos tem sido encontrada em outros locais no Brasil (PARDINI \& Trajano, 1999; KasPer et al., 2008; Quadros, 2012).

A espécie parece ter preferência por alguns abrigos específicos em vista da diferença na intensidade de uso entre eles. Esta preferência tem sido relatada para Lontra longicaudis em diversos locais da sua área de ocorrência (PARDini \& TRAJANO, 1999; WALDEMARIN \& Colares, 2000; Quadros \& Monteiro-Filho, 2002; NAKANO-Oliveira et al., 2004; KASPER et al., 2004; KASPER et al., 2008). A preferência de utilização dos abrigos na área estudada parece estar relacionada com a estrutura do mesmo, já que o abrigo utilizado de forma intensa era o único que se encontrava abaixo do nível 
do solo, desta forma, oferecendo maior proteção ao indivíduo em comparação aos abrigos acima do solo. No entanto, outros fatores tal como acessibilidade, oferta de alimento nas redondezas e proximidade com afluentes já foram apontados (KASPER et al., 2008; QUADROS, 2012) e podem também estar influenciando esta preferência.

Lontra longicaudis usou a extensão da área estudada de forma distinta, sendo que os sítios de marcação mais intensamente utilizados foram aqueles que se encontravam na proximidade dos abrigos. A associação entre abrigos e sítios de marcação também foi verificada por QUADros \& MONTEIRO-FILHO (2002) e QuADros (2012) que atribuem tal fato a uma possível demarcação e defesa de território. Diversos estudos corroboraram os abrigos e suas redondezas como o centro de atividade para outras espécies de lontras, confirmando também a relação da intensidade de marcação com a intensidade de uso de áreas específicas (Melquist \& Hornocker, 1983; Ruiz-Olmo et al., 2001; Mason \& MacDonald, 2009; QuAdros, 2012).

A inexistência de diferenças no uso do espaço entre a margem protegida em relação à margem fora de proteção demonstra certa tolerância da espécie a distúrbios humanos, que vai de acordo com o verificado por Soldateli \& Blacher (1996), Pardini \& Trajano (1999), Alarcon \& Simões-Lopes (2003) e Kasper et al. (2008). Estudos sugerem que a lontra pode ter tolerância a ações humanas dependendo do tamanho da área alterada e da presença de áreas preservadas na sua proximidade (PARDINI \& TRAJANO, 1999; KASPER et al., 2008); assim, a margem preservada pode estar influindo positivamente na presença da lontra nas áreas fora de proteção.

Apesar de não ter havido diferenças na utilização das duas margens, a intensidade de uso dos sítios de marcação esteve relacionada à cobertura vegetal do local, sendo os sítios mais utilizados localizados em áreas com maior cobertura, resultado este que corrobora com diversos estudos que identificaram que Lontra longicaudis utiliza com mais intensidade áreas de floresta preservada em outros locais do país, usando mais intensamente trechos do rio que possuem vegetação mais densa (WALDEMARIN \& COLARES, 2000; QuADRos \& Monteiro Filho, 2002; Alarcon \& Simões-Lopes, 2003; Quintela et al., 2011). No entanto, a cobertura vegetal explica apenas parcialmente o uso do espaço pela lontra, tendo em vista de que a correlação entre cobertura e marcação foi baixa. Outros fatores, tal como a localização dos abrigos, já aqui abordado, assim como outros não contemplados no presente estudo, podem também estar influenciando no padrão de uso do espaço pela lontra. Outras contribuições citaram fatores como proximidade com afluentes de grande porte e com foz de afluentes (QUADROS, 2012), o grau do distúrbio humano e a presença de locais disponíveis para marcação e abrigo (ALARCON \& SimÕEs-LOPES, 2003), evidenciando a complexidade do tema.
Concluindo, o uso do espaço pela espécie esteve relacionado à localização de seus abrigos, ao grau de cobertura vegetal do local e parece ter sido pouco influenciado pelo distúrbio humano. Ressalta-se a importância de novos estudos que avaliem outros fatores que possam estar influindo no uso do espaço pela lontra neotropical em locais alterados. Através dos dados deste estudo, pode-se concluir que para medidas de conservação da lontra neotropical é importante preservar a vegetação marginal dos rios, manter áreas íntegras nas proximidades de zonas com distúrbio humano para a permanência da espécie no local, ressaltando-se que as áreas com abrigos devem ser o foco das ações de preservação, já que há indícios que estes sejam os locais mais utilizados dentro da área de vida da espécie.

Agradecimentos. À Fundação Zoobotânica do Rio Grande do Sul pelo apoio logístico, e em especial a Arlete Ieda Pasqualetto, Ricardo Aranha Ramos e Thiago Seffrin (Laboratório de Geoprocessamento/ $\mathrm{MCN}$ ), pelas análises de geoprocessamento e elaboração dos mapas; aos técnicos Cleodir José Mansan e Tomaz Vital Aguzzoli (MCN) pelo auxílio nas saídas de campo. À COPESUL, antiga gestora do Parque, e funcionários pelo apoio logístico durante a execução das campanhas de campo. Ao CNPq pelas bolsas de iniciação científica concedidas à primeira autora.

\section{REFERÊNCIAS BIBLIOGRÁFICAS}

Alarcon, G. G. \& Simões-Lopes, P. C. 2003. Preserved versus degraded coastal environments: a case study of the neotropical otter in the environmental protection area of Anhatomirim, southern Brazil. IUCN Otter Specialist Group Bulletin 20(1):6-18.

EisenberG, J. F. \& Redford, K. H. 1999. Mammals of the Neotropics, v. 3, The Central tropics: Ecuador, Peru, Bolivia, Brazil. Chicago, London, University of Chicago Press. 609p.

Emmons, L. H. \& FeER, F. 1997. Neotropical Rainforest Mammals: A field guide. 2ed. Chicago, London, University of Chicago Press. 307p.

FEPAM (Fundação Estadual de Proteção Ambiental Henrique LUIZ RoESSLER). 2010. Qualidade ambiental. Região Hidrográfica do Guaíba. Rio Caí. Disponível em: http:/www.fepam.rs.gov.br/ qualidade/qualidade cai/cai.asp. Acesso em: 27.01.2011.

Guter, A.; Dolev, A.; SAltz, A. \& Kronfeld-Schor, A. 2008. Using videotaping to validate the use of spraints as an index of Eurasian otter (Lutra lutra) activity. Ecological Indicators 8:462-465.

IBGE (Instituto Brasileiro DE ECONOMIA E Estatística). 1986. Levantamento de recursos naturais, v. 33 - Folha SH. 22. Porto Alegre, Rio de Janeiro. p. 541-632.

IUCN. 2013. IUCN Red List of Threatened Species. Version 2013.1. Disponível em: <www.iucnredlist.org > Acesso em: 30.08.2013.

Kasper, C. B.; Feldens, M. J.; Salvi, J. \& Grillo, H. C. Z. 2004 Estudo preliminar sobre a ecologia de Lontra longicaudis (Olfers) (Carnivora, Mustelidae) no Vale do Taquari, sul do Brasil. Revista Brasileira de Zoologia 21(1):65-72

Kasper, K. B.; Bastazini, V. A. G.; Salvi, J. \& Grillo, H. C. J. 2008. Trophic ecology and the use of shelters and latrines by the neotropical otter (Lontra longicaudis) in the Taquari Valley, Southern Brazil. Iheringia, Série Zoologia 98(4):469-474.

Mason, C. F. \& MacDonald, S. M. 1987. The use of spraints for surveying otter Lutra lutra populations: an evaluation. Biological Conservation 41:167-177.

2009. Otters: Ecology and Conservation. Cambridge, Cambridge University. 236p.

Melquist, W. E. \& Hornocker, M. G. 1983. Ecology of River Otters in West Central Idaho. Wildlife Monographs 83:3-60.

NaKano-Oliveira, E.; Fusco, R.; Santos, E. A. V. \& MonteiroFilHo, E. L. A. 2004. New information about the behavior of Lontra longicaudis (Carnivora: Mustelidae) by radio-telemetry. IUCN Otter Specialist Group Bullettin 21(1):31-35. 
OldHam, A. R. \& Black, J. M. 2009. Experimental tests of latrine use and communication by River Otters. Northwestern Naturalist 90(3):207-211.

Oliveira, G. G.; Saldanha, D. L. \& Guaselli, L. A. 2010. Espacialização e análise das inundações na bacia hidrográfica do Rio Caí/RS. Geociências 29(3):413-427.

Pardini, R. \& Trajano, E. 1999. Use of shelters by the neotropical river otter (Lontra longicaudis) in an Atlantic Forest stream, southeastern Brazil. Journal of Mammalogy 80(2):600-610.

QuAdros, J. 2012. Uso do habitat e estimativa populacional de lontras antes e depois da formação do reservatório de Salto Caxias, rio Iguaçu, Paraná, Brasil. Neotropical Biology and Conservation 7(2):97-107.

Quadros, J. \& Monteiro-Filho, L. A. 2002. Sprainting sites of the Neotropical otter, Lontra longicaudis, in an Atlantic Forest area of southern Brazil. Mastozoología Neotropical 9(1):39-46.

Quintela, F. M.; DA Silva, F. A.; Assis, C. L. \& Antunes, V. C. 2012. Data on Lontra longicaudis (Carnivora: Mustelidae) Mortality in Southeast and Southern Brazil. IUCN Otter Specialist Group Bulletin 29(1):5-8.

Quintela, F. M.; Ibarra, C. \& Colares, E. P. 2011. Utilização de abrigos e latrinas por Lontra longicaudis (Olfers, 1818) em um arroio costeiro na Área de Proteção Ambiental da Lagoa Verde, Rio Grande do Sul, Brasil. Neotropical Biology and Conservation 6(1):35-43

Ruiz-Olmo, J.; SAaVEDRA, D. \& JimÉnez, J. 2001. Testing the surveys and visual and track censuses of Eurasian otters (Lutra lutra). Journal of Zoology 253:359-369.

Soldateli, M. \& BlaCher, C. 1996. Considerações preliminares sobre o número e distribuição espaço/temporal de sinais de Lutra longicaudis (Olfers, 1818) (Carnivora: Mustelidae) nas lagoas da Conceição e do Peri, Ilha de Santa Catarina, SC, Brasil. Biotemas 9(1):38-64.

Veitenheimer-Mendes, I. L.; Mondin, C. A. \& Strehl, T. 2000. Guia ilustrado de fauna e flora. 2ed. Porto Alegre, COPESULFZB. 209p.

Waldemarin, H. F. \& Alvarez, R. 2008. Lontra longicaudis. In: IUCN Red List of Threatened Species. Version 2013.1. Disponível em: $<$ www.iucnredlist.org $>$. Acesso em: 30.08.2013.

Waldemarin, H. F. \& Colares, E. P. 2000. Utilization of resting sites and dens by the neotropical river otter (Lontra longicaudis) in the south of Rio Grande do Sul State, Southern Brazil. IUCN Otter Specialist Group Bulletin 17(1):14-19 